EXTENDED REPORT

\title{
Angiopoietin concentrations in diabetic retinopathy
}

\author{
J I Patel, P G Hykin, Z J Gregor, M Boulton, I A Cree
}

Br J Ophthalmol 2005;89:480-483. doi: 10.1136/bjo.2004.049940

See end of article for authors' affiliations

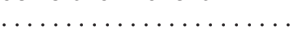

Correspondence to: Mr J I Patel, Department of Pathology, Institute of Ophthalmology, 11-43 Bath Street, London ECIV 9EL, UK; jigs37@hotmail. com

Accepted for publication 29 August 2004

\begin{abstract}
Background/aim: Angiopoietin 1 and 2 interact with vascular endothelial growth factor (VEGF) to promote angiogenesis in animal and in vitro models. Although VEGF concentrations are elevated, there is little information regarding angiopoietin concentration in the vitreous of patients with diabetic retinopathy. Methods: Angiopoietin concentrations were measured by luminescence immunoassay in vitreous samples from 17 patients with non-proliferative diabetic retinopathy (NPDR) and clinically significant diabetic macular oedema (CSMO), 10 patients with proliferative diabetic retinopathy (PDR), and five patients with macular hole (controls) obtained at pars plana vitrectomy.

Results: Angiopoietin 1 concentrations were low in patients with macular hole (median $17 \mathrm{pg} / \mathrm{ml}$ ) while in NPDR with CSMO they were 2002 pg/ml (range 289-5820 pg/ml) and in PDR 186 pg/ml (range 26$2292 \mathrm{pg} / \mathrm{ml}$ ). Angiopoietin 2 concentrations in NPDR with CSMO were a median of $4000 \mathrm{pg} / \mathrm{ml}$ (range 1341-14 $329 \mathrm{pg} / \mathrm{ml}$ ). For both macular hole and PDR patients angiopoietin 2 was below the limit of detection.

Conclusions: Angiopoietin 2 concentration was twice that of angiopoietin 1 in NPDR with CSMO. Angiopoietin 2 is the natural antagonist of angiopoietin 1 which is thought to act as an anti-permeability agent. The predominance of angiopoietin 2 may allow VEGF induced retinal vascular permeability in patients with CSMO. The relatively low concentration of both angiopoietin 1 and 2 in patients with proliferative diabetic retinopathy may reflect the established nature of the neovascularisation in cases proceeding to vitrectomy.
\end{abstract}

$\mathrm{T}$ he angiopoietins are growth factors that modulate the processes of physiological angiogenesis and pathological neovascularisation, ${ }^{12}$ particularly in association with vascular endothelial growth factor (VEGF)

Diabetic retinopathy is the commonest cause of blindness in the working age population. Its pathogenesis is increasingly understood with retinal ischaemia leading to neovascularisation and increased vascular permeability. ${ }^{3}$ How the angiopoietins interact in this process is unclear and their role in early diabetic retinopathy remains obscure.

There are four known angiopoietins (angiopoietins 1-4). ${ }^{4}$ Angiopoietins 1 and 2 have been studied in animal and in vitro models, particularly in relation to diabetic retinopathy. Angiopoietin 1 has been shown to promote endothelial cell survival without causing proliferation, ${ }^{5}$ and to stabilise endothelial interactions with surrounding support cells. ${ }^{6}$ It can block the vascular permeability effects of VEGF in vivo. ${ }^{67}$ In a rat diabetic model, angiopoietin 1 suppressed the development of diabetic retinopathy and reduced both vascular endothelial injury and blood-retinal barrier breakdown. ${ }^{8}$ In contrast, angiopoietin 2 is expressed at sites of vascular remodelling in the angiogenic process ${ }^{9}$ and is upregulated by hypoxia, VEGF, ${ }^{10}$ and retinal ischaemia ${ }^{11}$

Despite the evidence implicating angiopoietins 1 and 2 in the development of diabetic retinopathy, there are no established data on angiopoietins 1 and 2 concentrations in the ocular fluids of patients with diabetic retinopathy.

This study describes the concentrations of angiopoietins 1 and 2 in the ocular fluids of patients undergoing pars plana vitrectomy for either proliferative retinopathy or for clinically significant macular oedema in patients with non-proliferative retinopathy, compared with the concentration seen in patients with idiopathic macular hole (controls), and also correlate these concentrations with clinical parameters of the macula post-vitrectomy and how these concentrations may affect their interaction at the angiopoietin receptor, Tie- 2 . The patients with the macula hole represent normal non-angiogenic retina and the levels of angiopoietins in the vitreous would then contrast with the diabetic, ischaemic retina.

\section{PATIENTS AND METHODS}

Vitreous samples were collected from patients with idiopathic full thickness macular hole $(n=5)$, non-proliferative diabetic retinopathy (NPDR) with clinically significant macular oedema (CSMO) despite conventional ETDRS macular photocoagulation $(n=17)$, and treated proliferative diabetic retinopathy $(\mathrm{n}=10)$ undergoing standard three port pars plana vitrectomy. Patients with persisting diffuse CSMO also had optical coherence tomography (OCT) performed to assess the structural indices of the macula before and after surgery.

Inclusion criteria included (1) patients with idiopathic full thickness macular hole (as controls), (2) patients with nonproliferative diabetic retinopathy with persistent CSMO where (i) the CSMO involved the foveal centre for $<2$ years and (ii) and previous treatment with macular laser had been applied. Exclusion criteria were (i) posterior vitreous detachment diagnosed by the presence of a Weiss ring, (ii) macular traction as evidenced by retinal striae involving the foveal centre, (iii) macular ischaemia as defined by an enlarged foveolar avascular zone $(\mathrm{FAZ}>1000 \mu \mathrm{m})$ or significant perifoveal capillary loss on fundus fluorescein angiography (FFA), and (iv) co-existent retinal disease. Patients with proliferative retinopathy who had previously been treated with argon panretinal photocoagulation but developed fibrovascular tractional/rhegmatogenous retinal detachment were included in the proliferative group of patients. All

\footnotetext{
Abbreviations: BSA, bovine serum albumin; CSMO, clinically significant diabetic macular oedema; FAZ, foveolar avascular zone; FFA, fundus fluorescein angiography; FTMH, full thickness macular hole; LIA, luminescence immunoassay; NPDR, non-proliferative diabetic retinopathy; $\mathrm{OCT}$, optical coherence tomography; PBS, phosphate buffer saline; PDR, proliferative diabetic retinopathy; VEGF, vascular endothelial growth factor
} 
patients underwent standard three port pars plana vitrectomy with elimination and removal of the posterior vitreous cortex but without peeling of the internal limiting membrane. Patients were recruited in accordance with the Declaration of Helsinki and with the approval of the ethics committee of Moorfields Eye Hospital.

Postoperative aqueous samples taken at 2 and 6 weeks were also used to correlate changes in the angiopoietin concentration in the patients with NPDR and CSMO and the structural thickness of the macula after surgery where sufficient aqueous could be obtained for assay.

\section{Growth factor analysis}

Baseline vitreous (up to $500 \mu \mathrm{l}$ ) and aqueous (100 $\mu \mathrm{l}$ ) samples were taken immediately before the start of vitrectomy, and the postoperative aqueous ( $100 \mu \mathrm{l})$ samples were immediately placed in ice, centrifuged, and the supernatant divided into aliquots and stored at $-20^{\circ} \mathrm{C}$ until laboratory analysis. The levels of growth factors $(\mathrm{pg} / \mathrm{ml})$ were analysed using a luminescence immunoassay (LIA), designed for this purpose to use the very small volumes of ocular fluids available

A volume of $30 \mu \mathrm{l}$ recombinant FcTie-2 receptor (Regeneron Pharmaceuticals Inc) was added to wells of a 384 well ELISA plate and incubated overnight at $4^{\circ} \mathrm{C}$ at a concentration of $3 \mu \mathrm{g} / \mathrm{ml}$. The wells were then washed six times with $100 \mu \mathrm{l}$ of phosphate buffer saline (PBS) and then blocked with $100 \mu \mathrm{l}$ of PBS- $0.5 \%$ of bovine serum albumin (BSA) for 1 hour at room temperature. Then either $30 \mu \mathrm{l}$ of either angiopoietin $\mathrm{l}$ ( $\mathrm{R}$ and D Systems, Minneapolis MN, USA) or angiopoietin 2 (Regeneron Pharmaceuticals Inc, USA) standards, or test samples were added to the wells for 2 hours at room temperature (samples were diluted 1:4 with phosphate buffered saline, $\mathrm{pH}$ 7.4). The wells were then washed six times with PBS. Thereafter, either $30 \mu \mathrm{l}$ of NTL-1 (secondary antibody for angiopoietin 1) or NTL-2 (secondary antibody for angiopoietin 2) was added to each well depending on which cytokine was tested (Regeneron Pharmaceuticals Inc, USA). Both secondary antibodies were used at 1:10 000 in PBS-0.5\% BSA. The wells were then washed, and $30 \mu \mathrm{l}$ of a tertiary antibody of anti-rabbit goat immunoglobulin added at 1:10 000 for 1 hour at room temperature. Then the wells were washed with PBS followed by addition of $30 \mu \mathrm{l}$ of LumiGLO reagent and hydrogen peroxide (New England Biolabs, USA) for 5 minutes and the plate read in a luminometer. The angiopoietin l standard curve was from to $5 \mathrm{pg} / \mathrm{ml}-100 \mathrm{ng} / \mathrm{ml}$. All of the results of the samples fell within this range. Similarly, for angiopoietin 2, the standard curve ranged from $1 \mathrm{ng} / \mathrm{ml}$ to $100 \mathrm{ng} / \mathrm{ml}$. The lower sensitivity of the angiopoietin 2 assay is explained by the lower affinity of angiopoietin 2 for Tie 2 in comparison with angiopoietin 1. Vitreous samples were tested in triplicate and the aqueous samples tested in duplicate.

\section{OCT}

OCT was performed to allow correlation between the structural indices of the macula before and after surgery and angiopoietin levels. OCT is a non-invasive, non-contact imaging modality producing high resolution cross sectional tomographs of ocular tissue. ${ }^{12}$ It produces a two dimensional false colour image of the back scattered light from different layers of the retina analogous to ultrasonic B-scan imaging. The only difference is that optical reflectivity is measured. Axial resolution up to $14 \mu \mathrm{m}$ is achievable. Six $6 \mathrm{~mm}$ diameter radial scans centred on the point of fixation were taken at each time point, and assessed qualitatively and analysed quantitatively using the retinal mapping software. Quantitative assessment using the computer software (ZeissHumphrey, Dublin, CA, USA, software version A5) included
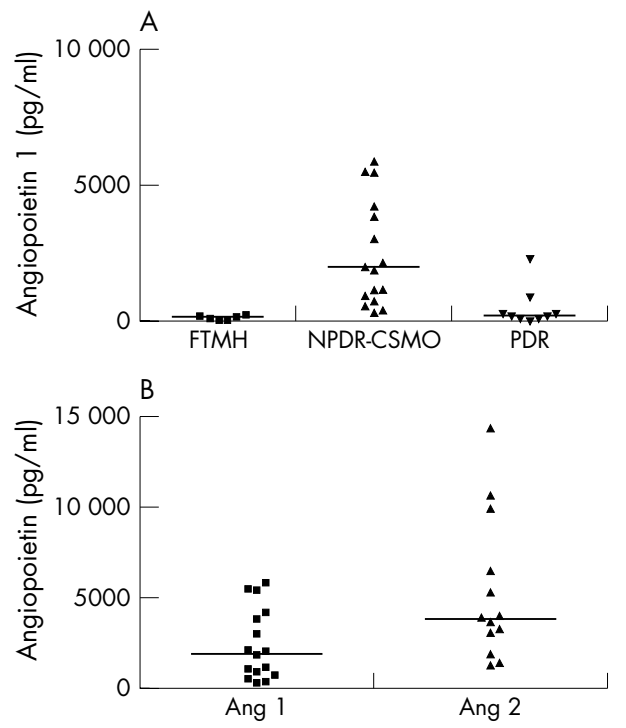

Figure 1 (A) Vitreous angiopoietin 1 concentration in full thickness macular hole (FTMH) $(n=5)$, non-proliferative diabetic retinopathy with clinically significant macular oedema (NPDR-CSMO) $(n=17)$, and treated proliferative diabetic retinopathy (PDR) $(n=10)$. The angiopoietin 1 concentration was greater in the NPDR than in FTMH and PDR $(p=<0.001)$. (B) Vitreous angiopoietin concentration (Ang 1 and Ang 2) in non-proliferative diabetic retinopathy with clinically significant macular oedema. Angiopoietin 1 concentration was approximately half that of angiopoietin 2 .

an estimation of the foveal thickness and the macular volume, which is derived by an integration process of the average retinal thickness of the six radial scans.

\section{Statistical analysis}

Mann-Whitney test and non-parametric (Spearman) correlation tests were used to analyse the data.

\section{RESULTS}

The average duration of diabetes was 14 years (5-22 years) with $\mathrm{HbA}_{1 \mathrm{C}}$ of $9 \%(9-12 \%)$ in the diabetic patients whose average age was 58 (40-72 years). All had type 2 diabetes. Those patients with non-proliferative retinopathy also demonstrated clinically significant macular oedema which had been present for a median of 15 months (12-18 months) before pars plana vitrectomy. They had received on average three macular laser treatments (range 1-6). Those with proliferative disease had received extensive panretinal photocoagulation and underwent PPV for delamination of membranes and further endolaser.

The median vitreous angiopoietin 1 concentration for five patients with full thickness macular hole (FTMH) used as a control population was $150 \mathrm{pg} / \mathrm{ml}$ (range 3-227 pg/ml) compared to $1933 \mathrm{pg} / \mathrm{ml}$ (range $289-5820 \mathrm{pg} / \mathrm{ml}$ ) in 17 patients with non-proliferative diabetic retinopathy (NPDR) and $186 \mathrm{pg} / \mathrm{ml}$ (range 26-2,292 pg/ml) for 10 patients with treated proliferative diabetic retinopathy (PDR) (see fig lA). The angiopoietin 1 concentration in NPDR were higher than in FTMH $(\mathrm{p}<0.001)$ and PDR $(\mathrm{p}<0.01)$ while there was no difference in the angiopoietin 1 concentration between PDR and FTMH (NS).

The median angiopoietin 2 concentration in NPDR was $3874 \mathrm{pg} / \mathrm{ml}$ (range 134l-9888 pg/ml) while in FTMH and PDR patients, angiopoietin 2 concentration were below the level of sensitivity for the assay. The angiopoietin 1 concentration in NPDR was half that of angiopoietin 2 with the difference significant at $\mathrm{p}<0.02$ (see fig lB). 


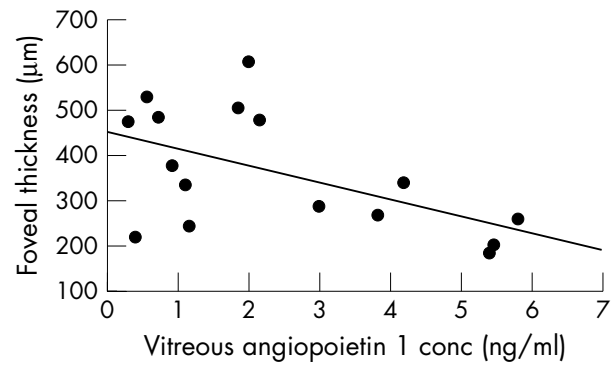

Figure 2 Inverse relation between vitreous angiopoietin 1 and foveal thickness. ( $R=-0.44, p=0.08$ Spearman correlation test) in the NPDRCSMO patients.

Further on comparing the foveal thickness to the angiopoietin 1 concentration in patients with NPDR, all of whom had persistent CSMO, there was a non-significant trend towards correlation $(\mathrm{p}<0.08$ and $\mathrm{R}=0.44$ ) ( see fig 2$)$. No such trend was seen on comparing angiopoietin 2 with these macular structural indices.

In two patients there was sufficient postoperative aqueous available (initially drawn as part of a separate study) to evaluate postoperative aqueous angiopoietin concentration over a 6 week period. This concentration varied according to whether there was a coincidental increase or decrease in the foveal thickness after surgery. In one patient there was a fall in the foveal thickness, with the angiopoietin 1 concentration increasing from a baseline of $845 \mathrm{pg} / \mathrm{ml}$ to $3839 \mathrm{pg} / \mathrm{ml}$ at 6 weeks as the foveal thickness changed from $216 \mu \mathrm{m}$ to $260 \mu \mathrm{m}$ over the same period, but at 3 months the thickness had decreased to $174 \mu \mathrm{m}$ (fig 3 ). The angiopoietin 2 level remained relatively static ( 3223 to $3591 \mathrm{pg} / \mathrm{ml}$ over the period of observation) (fig 3). However, in the second patient, the foveal thickness increased and the angiopoietin 1 concentration decreased over the 6 week period ( 2752 to $1828 \mathrm{pg} / \mathrm{ml}$ ) while angiopoietin 2 showed a reciprocal increase from 3069 to $6079 \mathrm{pg} / \mathrm{ml}$ over the same period (see fig 4).

\section{DISCUSSION}

Diabetic macular oedema is the leading cause of blindness among the diabetic population. ${ }^{13}$ Its pathogenesis is multifactorial and incompletely understood, but chronic
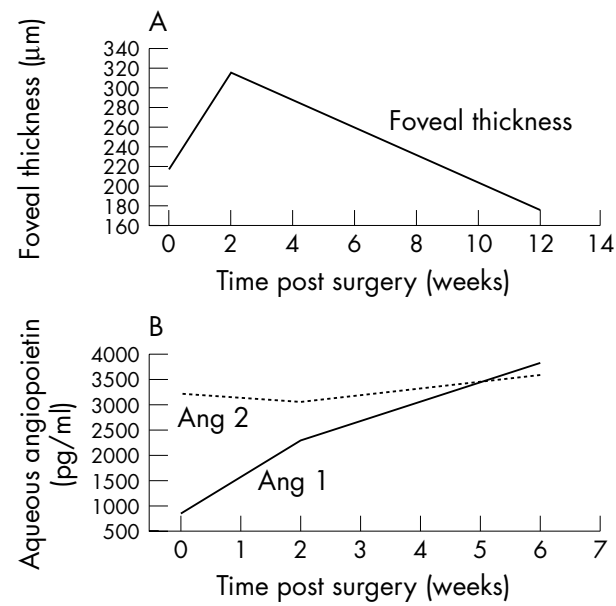

Number of weeks $v$ angiopoietin 1 Number of weeks $v$ angiopoietin 2

Figure 3 Correlation of foveal thickness with aqueous angiopoietin concentrations after pars plana vitrectomy. Reduced foveal thickness (A) corresponded with an increase in angiopoietin 1 concentration (B).
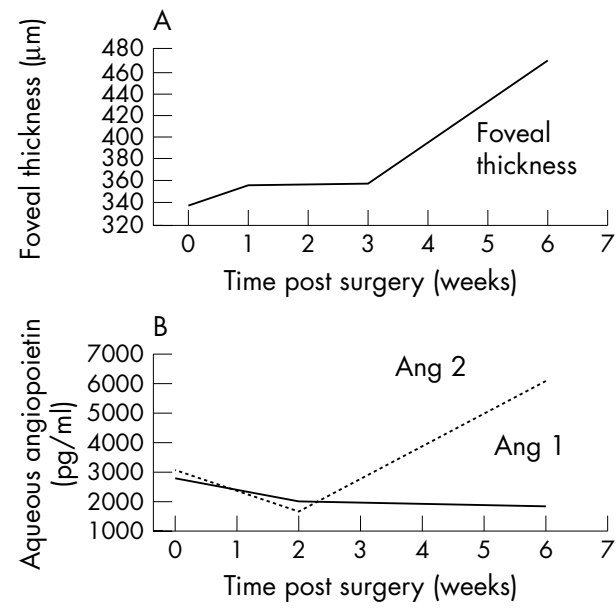

- Number of weeks $v$ angiopoietin 1 ........ Number of weeks $v$ angiopoietin 2

Figure 4 Correlation of foveal thickness with aqueous angiopoietin concentrations after pars plana vitrectomy. Increased foveal thickness (A) corresponded with increased angiopoietin 2 and reduced angiopoietin 1 concentration (B).

hyperglycaemia has been shown to disrupt the blood-retinal barrier $^{14}$ allowing for the development of oedema. Cytokines and growth factors are considered important in the process of damage to the blood-retinal barrier, in particular vascular endothelial growth factor (VEGF). VEGF is a vasopermeability inducing agent with known disruptive effects on retinal endothelial tight junctions. ${ }^{15}$ VEGF has been shown to work in concert with the angiopoietin ${ }^{16}$ which interact with the Tie 2 receptor. To date only concentrations of VEGF in the ocular fluids of diabetic patients are known. ${ }^{3}$ This is the first report of the concentration of angiopoietins in the vitreous of diabetic patients.

In PDR, the vitreous angiopoietin 1 level was similar to those of macular hole (FTMH) patients. The angiopoietin 2 concentration was below the sensitivity of the assay for both FTMH and PDR. This finding reflects the role of angiopoietin 2 in facilitating the process of angiogenesis at sites of active remodelling. ${ }^{9}$ Therefore, if there is no active angiogenesis (as in FTMH), the angiopoietin 2 level would not be elevated. Our results in PDR vitreous samples appear to contradict the role of angiopoietin 2 as a facilitator of angiogenesis, but may simply reflect the fact that in long standing and treated PDR, there is little active vessel replication. In established PDR, at the time of vitrectomy, new vessels are mature with limited active angiogenesis and therefore low concentrations of the angiopoietins would be expected. Furthermore in such a clinical picture of minimal vascular turnover, VEGF levels would also be at basal levels ${ }^{17}$ and, correspondingly, a decrease stimulus for angiopoietin 2 production. ${ }^{10}$ In FTMH no active neovascular processes are known to occur, explaining the minimal concentration of angiopoietin 1. In fact, FTMH patients represent the normal non-neovascular vitreoretinal environment where angiopoietin $\mathrm{l}$ is at the basal level required to promote and maintain endothelial integrity. In contrast, angiopoietin 1 promotes stabilisation of immature neovascularisation by promoting the recruitment of supporting cells. ${ }^{18}$ In treated PDR, where neovascularisation would be regressing, then angiopoietin 1 may be expected to contribute to this process as facilitator for the recruitment of supporting cells-for example, fibroblasts. There, in both FTMH and treated PDR similar levels of angiopoietin 1 may be expected. 
In contrast, patients with NPDR had angiopoietin 1 vitreous concentrations that were approximately half those of angiopoietin 2. In our series, patients had at least moderate and five cases had severe NPDR. From clinical observation there is established retinal ischaemia and capillary closure in such cases leading to the formation of IRMA (intraretinal microvascular abnormalities-precursors of new vessels) as a result of increased VEGF production. ${ }^{19}$ This represents the point in the natural history of diabetic retinopathy where early neovascularisation occurs with the development of angiogenic sites and endothelial remodelling. Such dynamic changes within the retina are probably reflected by the increased angiopoietin 2 concentration combined with a relative change in the angiopoietin 1 to angiopoietin 2 ratio allowing angiopoietin 2 to predominate at the Tie 2 receptor. The affinities of these two angiopoietins are similar for the Tie 2 receptor $^{18}$ but angiopoietin 2 competitively inhibits angiopoietin 1 at the receptor.9 Our results confirm that in NPDR, angiopoietin 2 concentration is double that of angiopoietin 1 concentration. Therefore the predominance of angiopoietin 2 at the Tie 2 receptor would promote increased vascular permeability in combination with elevated VEGF in NPDR ${ }^{20}$ thus facilitating breakdown of the bloodretinal barrier.

Interestingly, our findings in two patients who had intraoperative and two postoperative aqueous samples concur with the role of angiopoietin 2 as a pro-permeability agent. In the one patient, in whom the foveal thickness had deceased at 3 months after surgery, the angiopoietin 1 concentration had progressively increased during the initial 6 week period following surgery. We postulate that in this case, the antipermeability effect of angiopoietin 1 acted to reduce the amount of macula oedema as measured by OCT. In the second patient in which the postoperative macular oedema increased up to 3 months, there was a concomitant reduction in angiopoietin 1 aqueous concentration leading to increased permeability and macular oedema mediated by angiopoietin 2 (probably with VEGF). It seems that the morphological effects of the early changes in angiopoietin 1 after surgery may be borne out some weeks later. If this model of angiopoietin 1 function is correct, then an increase in angiopoietin 1 in this situation would precipitate a cascade of intracellular events which would eventually lead to the "tightening" of the blood-retinal barrier. ${ }^{21}$ However, a larger study correlating macular volume, as measured by OCT, and postoperative aqueous angiopoietin concentrations would be required to confirm these findings.

This is the first report to quantify the concentration of the angiopoietins 1 and 2 in the ocular fluids of diabetic patients and controls. It shows that both angiopoietin 1 and 2 are elevated in moderate and severe NPDR at a time when intraretinal neovascularisation is initiated. It is presumed that angiopoietin 2 predominates in this context to promote neovascularisation and possibly increased vascular permeability together with a decrease in angiopoietin 1 to precipitate macular oedema

\section{Authors' affiliations}

J I Patel, P G Hykin, Z J Gregor, Moorfields Eye Hospital, London, UK J I Patel, I A Cree, Institute of Ophthalmology, London, UK

M Boulton, School of Optometry and Vision Sciences, Cardiff University, Cardiff, UK

\section{REFERENCES}

1 Adamis AP, Shima D, Tolentino $M$, et al. Inhibition of VEGF prevents retinal ischaemia-associated iris neovascularization in a primate. Arch Ophthalmol 1996;114:66-71.

2 Sato TN, Tozawa H, Deutsch U, et al. Distinct roles of receptor tyrosine kinases Tie-1 and Tie-2 in blood vessel formation. Nature 1995;376:70-4.

3 Aiello LP, Avery R, Arrigg P, et al. Vascular endotheilal growth factor in ocular fluid of patients with diabetic retinopathy and other retinal disorders. N Engl J Med 1994;331:1480-7.

4 Koh GY, Kim I, Kwak HJ, et al. Biomedical significance of endothelial cell specific growth factor, angiopoietin. Expl Mol Med 2002;34:1-11.

5 Kim I, Moon S, Park SK, et al. Angiopoietin-1 regulates endothelial cell survival through the phosphatidylinositol $3^{\prime}$-kinase/Akt signal transduction pathway. Circ Res 2000;86:24-9.

6 Thurston G, Suri C, Smith K, et al. Leakage-resisitant blood vessels in mice trangenically overexpressing angiopoietin-1. Science 1999;286:2511-14.

7 Thurston G, Rudge J, loffe E, et al. Angiopoietin-1 protects the adult vasculature against plasma leakage. Nat Med 2000;6:460-3.

8 Joussen A, Poulaki V, Tsujikawa A, et al. Suppression of diabetic retinopathy with angiopoietin 1. Am J Pathol 2002;160:1683-93.

9 Maisonpierre PC, Suri C, Jones PF, et al. Angiopoietin-2, a natural antagonist for Tie 2 that disrupts in vivo angiogenesis. Science 1997;277:55-60.

10 Mandriota SJ, Pepper M. Regulation of angiopoietin-2 mRNA levels in bovine microvascular endothelial cells by cytokines and hypoxia. Circ Res 1998;83:852-9.

11 Oh H, Takagi H, Suzuma K, et al. Hypoxia and vascular endothelial growth factor selectively up-regulate angiopoietin-2 in bovine microvascular endothelial cells. J Biol Chem 1999;274:15732-9.

12 Huang D, Swanson E, Lin C, et al. Optical coherence tomography. Science 1991;254:1178-81

13 Clark J, Grey R, Lim K, et al. Loss of vision before ophthalmic referral in blind and partially sighted diabetics in Bristol. Br J Ophthalmol 1994;78:741-4.

14 Do Carmo A, Ramos P, Reis A, et al. Breakdown of the inner and outer blood reinal barrier in streptozotocin-induced diabetes. Exp Eye Res 1998;67:569-75.

15 Antonetti D, Barber A, Hollinger L, et al. Vascular endothelial growth factor induces rapid phosphorylation of tight junction proteins occludin and zonula occluden 1. A potential mechanism for vascular permeability in diabetic retinopathy and tumors. J Biol Chem 1999;274:23463-7.

16 Asahara T, Chen D, Takahashi T, et al. Tie 2 receptor ligands, angiopoietin-1 and angiopoietin-2, modulate VEGF-induced postnatal neovascularization. Circ Res 1998;83:233-40.

17 Aiello L, Avery R, Arrigg P, et al. Vascular endotheilal growth factor in ocular fluid of patients with diabetic retinopathy and other retinal disorders. N Engl J Med 1994;331:1480-7.

18 Suri C, Jones P, Patan S, et al. Requisite role of angiopoietin-1, a ligand for the Tie-2 receptor, during embryonic angiogenesis. Cell 1996;87:1171-80.

19 Murata T, Nakagawa K, Khalil A, et al. The relation between expression of vascular endothelial growth factor and breakdown of the blood-retinal barrier in diabetic rat retinas. Lab Invest 1996;74:819-25.

20 Murata T, Ishibashi T, Khalil A, et al. Vascular endothelial growth factor plays a role in hyperpermeability of diabetic retinal vessels. Ophthalmic Res 1995;27:48-52.

21 Gamble JR, Drew J, Trezise L, et al. Angiopoietin-1 is an antipermeability and anti-inflammatory agent in vitro and targets cell junctions. Circ Res 2000;87:603-7. 